\title{
An exploratory study of contractile force production in muscle fibers from patients with inflammatory myopathies
}

\author{
Franclo Henning MD, $\mathrm{PhD}^{1,2}$ @ | Tertius Abraham Kohn $\mathrm{PhD}^{2,3}$
}

\author{
${ }^{1}$ Division of Neurology, Department of \\ Medicine, Faculty of Medicine and Health \\ Sciences, Stellenbosch University, Cape Town,, \\ South Africa \\ ${ }^{2}$ Division of Exercise Science and Sports \\ Medicine, Department of Human Biology, \\ University of Cape Town, Newlands, \\ South Africa \\ ${ }^{3}$ Department of Medical Bioscience, Faculty of \\ Natural Sciences, University of the Western \\ Cape, Cape Town, South Africa

\section{Correspondence} \\ Franclo Henning MD, PhD, Division of \\ Neurology, Faculty of Medicine andHealth \\ Sciences, Stellenbosch University, PO Box \\ 19063, Tygerberg, 7505, CapeTown, South \\ Africa, \\ Email: fhenning@sun.ac.za

\section{Funding information} \\ South African Agency for Science and \\ Technology Advancement, Grant/Award \\ Number: 81837; Harry Crossley Foundation, \\ Grant/Award Number: SU-PT-15/09-000090
}

\begin{abstract}
Introduction: The mechanism by which weakness develops in idiopathic inflammatory myopathies (IIMs) is still unclear. In this study we investigated the maximum force of single muscle fibers from patients with IIMs.

Methods: Permeabilized single muscle fibers from patients with IIMs and healthy controls were subjected to contractility measurements. Maximum force and specific force production (maximum force normalized to fiber size) and fiber type were determined for each isolated fiber.

Results: A total of 178 fibers were studied from five patients with IIMs and 95 fibers from four controls. Specific force production was significantly lower in the IIM group for all fiber types.

Discussion: The findings from this exploratory study suggest that weakness in IIMs may, in part, be caused by dysfunction of the contractile apparatus. These findings provide a basis for further studies into the mechanisms underlying weakness in IIMs.

\section{KEYWORDS}

dermatomyositis, immune-mediated muscle disease, inflammatory myopathies, necrotizing autoimmune myopathy, polymyositis, single-fiber contractility
\end{abstract}

\section{1 | INTRODUCTION}

Idiopathic inflammatory myopathies (IIMs) are autoimmune muscle disorders of unknown cause that include dermatomyositis (DM), polymyositis (PM), necrotizing autoimmune myopathy (NAM), and inclusion-body myositis (IBM). In contrast to many hereditary myopathies, where abnormal or dysfunctional proteins may lead to impaired muscle fiber contractility, ${ }^{1-3}$ the mechanism of weakness in IIMs is less clear. Weakness is unlikely to be due solely to the loss of fibers from inflammatory necrosis, as the degree of inflammation and muscle weakness do not correlate, ${ }^{4}$ the number of necrotic fibers from histological analysis is usually small, ${ }^{5-8}$ and typically a rapid improvement in strength follows treatment with corticosteroids. ${ }^{6,7}$ Theoretically, weakness results from either a decrease in fiber numbers or impaired

Abbreviations: CSA, cross-sectional area; DM, dermatomyositis; IBM, inclusion-body myositis; IIM, idiopathic inflammatory myopathy; MRC, Medical Research Council; NAM, necrotizing autoimmune myopathy; $\mathrm{P}_{0}$, maximum force; $\mathrm{PM}$, polymyositis; $\mathrm{SF}$, specific force; TNF- $\alpha$, tumor necrosis factor-alpha. contractile function of individual fibers. We hypothesized that inflammation leads to impaired contractile function, and employed in vitro permeabilized single-muscle-fiber contractility studies to assess contractile function at a cellular level in IIM patients and healthy controls.

\section{2 | METHODS}

\section{1 | Muscle biopsies}

Adult participants with suspected IIM and who were immunosuppressive treatment-naive were recruited at Tygerberg Academic Hospital, Cape Town, South Africa, and referred to undergo diagnostic muscle biopsies. Only tissue from participants with a confirmed diagnosis of IIM, based on accepted criteria, ${ }^{9,10}$ and who had responded to corticosteroid treatment (initiated after the muscle biopsy) at 6 weeks after initiation, was included in the study. Patients with IBM were excluded, due to the different pathogenesis, course, time to diagnosis, and 
response to treatment. ${ }^{11-13}$ Controls consisted of healthy adults who donated muscle samples for research at the University of Cape Town Research Unit for Exercise Science and Sports Medicine. The human research ethics committees of both Stellenbosch University and the University of Cape Town approved the study and participants provided informed consent.

Muscle biopsies were performed under local anesthesia and were taken from the vastus lateralis muscle, 2 to $3 \mathrm{~cm}$ anterior to the midpoint of a line connecting the greater trochanter and the superior patellar margin. In controls, biopsies were performed using a Bergström needle via a 5- to 7-mm incision. In patients with IIM, open biopsies were performed to ensure acquisition of sufficient tissue for diagnostic purposes, and small segments of muscle were allocated to this study. Fresh muscle specimens were divided into two or three samples of approximately $6 \times 4 \times 4 \mathrm{~mm}$, rapidly frozen in liquid nitrogen, and stored at $-200^{\circ} \mathrm{C}$ until analysis. Before analysis, each stored sample was thawed briefly in phosphate-buffered saline at $37^{\circ} \mathrm{C}$ for 1 minute and divided into smaller bundles, each consisting of 20 to 40 fibers. These bundles were then submerged into skinning solution containing $50 \%$ glycerol $(\mathrm{pH} 7.00)$ and stored at $4{ }^{\circ} \mathrm{C}$ for 24 hours, replaced with fresh skinning solution the following day, and then stored at $-20^{\circ} \mathrm{C}$ until analysis. ${ }^{14}$

\subsection{Contractility studies}

Contractile properties of skinned single fibers were analyzed as previously described using a permeabilized single-fiber test system (Aurora Scientific, Ontario, Canada). ${ }^{15}$ Cross-sectional area (CSA) was determined from the diameter of the fiber using the equation $\pi[(0.8 \times$ fiber diameter) / 2 $]^{2}$, where 0.8 is to correct for an estimated $20 \%$ fiber swelling. ${ }^{16}$ Absolute force was measured in milli-newtons, and specific force (SF) was calculated as maximum force $\left(P_{0}\right)$ normalized to CSA and expressed as kilo-newtons per meter squared. All experiments were performed at $12^{\circ} \mathrm{C}$. The myosin heavy chain composition of each fiber was individually determined with gel electrophoresis and silver staining. ${ }^{17}$

\section{3 | Statistical analysis}

Statistical comparisons between IIMs and controls were performed for all fiber types combined (types I, IIA, IIX, and hybrid), and for type I and IIA fibers separately. Type IIX and hybrid fibers were not compared separately due to their absence or small numbers in the biopsies. The D'Agostino-Pearson normality test was used to test for normality of distribution. The unpaired $t$ test with Welch's correction was used to compare means and medians when data sets displayed a normal distribution, whereas the nonparametric Mann-Whitney $U$ test was used for data sets with non-normally distributed data. Statistical analysis was performed using GraphPad Prism version 7 (GraphPad Software, La Jolla, California). For all parameters, mean \pm standard deviation (SD) and median with interquartile range were calculated. Statistical significance was set at $P<.05$.

\section{3 | RESULTS}

Participants included consisted of four healthy controls (all females; mean age, 28 years) and five patients with IIMs (all females; mean age, 48 years). The median knee extension Medical Research Council (MRC) strength score for the IIM group was 4 (Table 1). Select muscle histology images are shown in Figure S1 online.

\section{1 $\quad$ CSA, $P_{0}$, and SF}

Overall, 178 fibers were studied from patients with IIMs, amounting to 55 type I, 98 type IIA, 6 type IIX, and 19 hybrid fibers (13 I/IIA, 2 I/IIX, 4 IIA/IIX). A total of 95 fibers, consisting of 59 type I, 29 type IIA, and 7 hybrid fibers (all I/IIA), were studied from the healthy control group.

Combined, there was no difference in mean CSA between the IIM and control groups. When compared separately, mean type I fiber CSA was 9\% smaller in the IIMs group compared with controls, whereas mean type IIA fiber CSA was $24 \%$ smaller in the control

TAB LE 1 Demographic and clinical characteristics of participants ${ }^{a}$

\begin{tabular}{lllll} 
Participant & Diagnosis & Age (years) & $\begin{array}{l}\text { MRC muscle strength grade } \\
\text { for knee extension }\end{array}$ & $\begin{array}{l}\text { Modified Rankin } \\
\text { Scale score }\end{array}$ \\
\hline IIM1 & DM & 42 & 5 & 3 \\
\hline IIM2 & PM & 60 & 4 & 4 \\
\hline IIM3 & DM & 58 & 4 & 1 \\
\hline IIM4 & NAM & 26 & 4 & 4 \\
\hline IIM5 & NAM & 53 & $4^{-}$ & 0 \\
\hline HC1 & HC & 22 & Not tested & 0 \\
\hline HC2 & HC & 44 & Not tested & 0 \\
\hline HC3 & HC & 27 & Not tested & 0 \\
\hline HC4 & HC & 20 & Not tested & \\
\hline
\end{tabular}

Abbreviations: DM, dermatomyositis; HC, healthy control; IIM, idiopathic inflammatory myopathy; MRC, Medical Research Council; NAM, necrotizing autoimmune myopathy; PM, polymyositis.

${ }^{a}$ All females. 
TAB LE 2 CSA, $P_{0}$, and SF for IIM cases and female controls

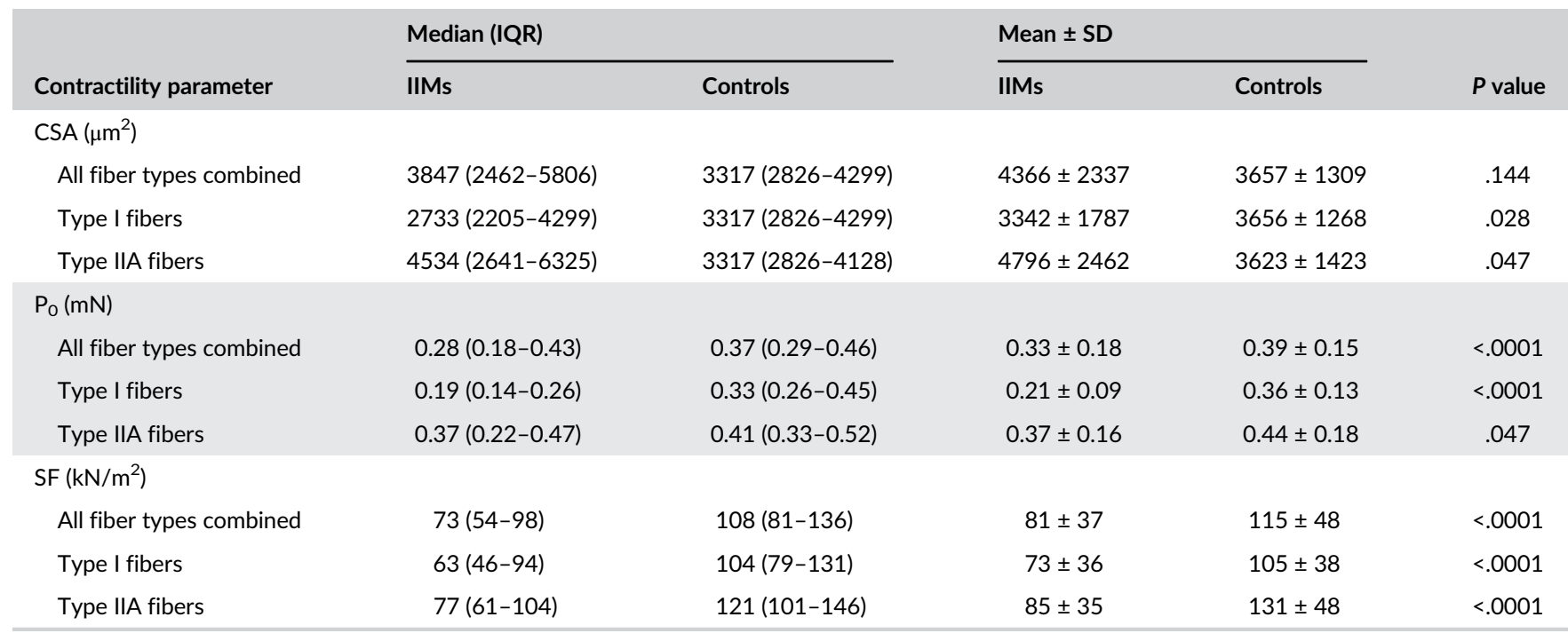

Abbreviations: CSA, cross-sectional area; IIM, inflammatory myopathy; IQR, interquartile range; $\mathrm{P}_{0}$, maximum force; SF, specific force.
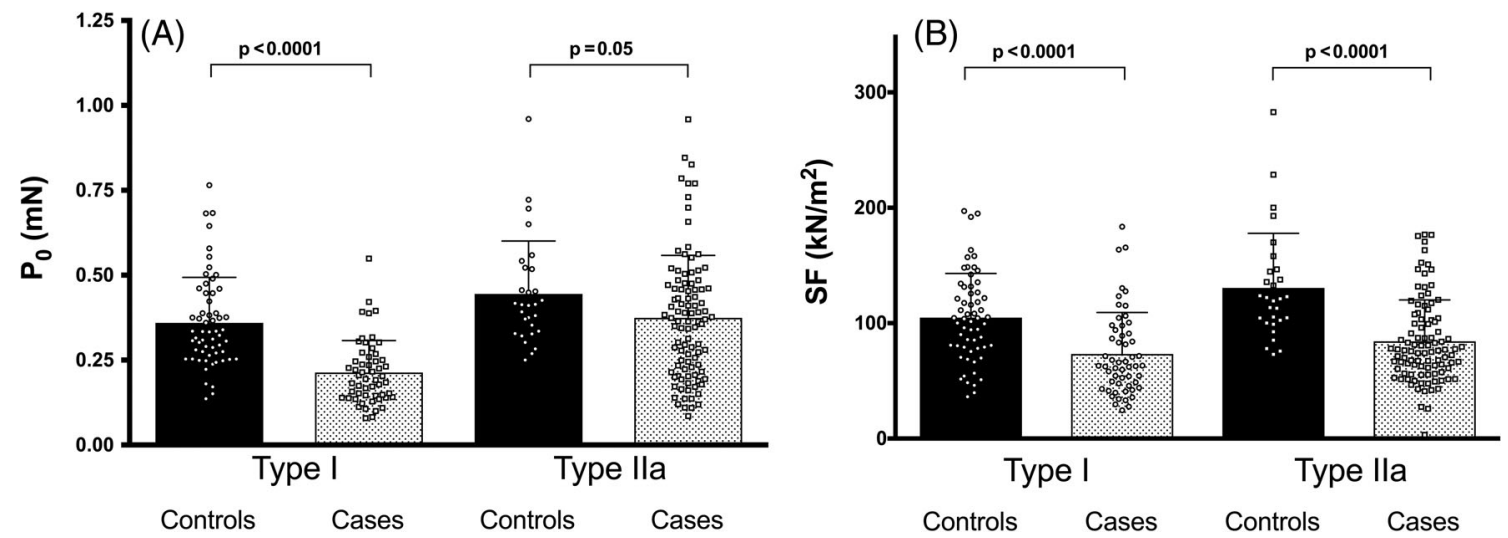

FIGURE 1 Maximum force (A) and specific force (B) of type I and IIA fibers from controls and idiopathic inflammatory myopathy cases. $\mathrm{P}_{0}$, maximum force; $\mathrm{SF}$, specific force

group (Table 2). Combined, $\mathrm{P}_{\mathrm{O}}$ and SF were $15 \%$ and $30 \%$ lower in the IIMs group, respectively. $\mathrm{P}_{0}$ and SF of type I fibers were $42 \%$ and $30 \%$ lower in the IIMs group, whereas $\mathrm{P}_{0}$ and SF of type IIA fibers were $16 \%$ and $35 \%$ lower (Figure 1 and Table 2).

\section{4 | DISCUSSION}

The findings of this study suggest that contractile force is impaired in IIMs, which is independent of a decrease in fiber size, as evidenced by decreased SF after correcting for CSA. The implication of this finding is that muscle weakness in IIMs is related to a functional impairment of muscle fiber contraction, and not a decrease in fiber size.

A number of different mechanisms have been proposed to explain weakness and fatigue in IIMs, including impaired sarcoplasmic reticulum $\mathrm{Ca}^{2+}$ release ${ }^{18}$ and abnormalities in energy metabolism, such as adenosine monophosphate deaminase 1 (AMPD1) deficiency. ${ }^{4}$ However, we also observed impaired contractility, although $\mathrm{Ca}^{2+}$ and adenosine triphosphate were provided in sufficient quantities by the activating solution, thus discrediting abnormal $\mathrm{Ca}^{2+}$ release and energy metabolism.

A possible explanation for the impaired contractility may involve an interaction between one or more components of the inflammatory response and the contractile apparatus or its supporting structures. One such component is tumor necrosis factor-alpha (TNF- $\alpha$ ), which has been shown to decrease contractile force of skeletal muscles in animal models. ${ }^{19-22}$ in vivo and in vitro experiments utilizing dog, hamster, and mouse models have shown impaired contractility within hours after TNF- $\alpha$ administration or incubation, both at the muscle (diaphragm) and myofibrillar level. The effect of TNF- $\alpha$ was partially blocked by the cyclooxygenase inhibitor indomethacin and by trolox (an antioxidant), suggesting that the action of TNF- $\alpha$ is mediated by cyclooxygenase products and/or intracellular oxidant activity. ${ }^{21,22}$

Other than the effect of TNF- $\alpha$, different explanations for the decrease in contractility should be considered. One such possibility is an 
indirect effect of the disease process on force production, mediated by muscle disuse and inactivity. The effects of both long- and short-term immobilization have been investigated, and have been shown to decrease single-fiber force production in some, ${ }^{23-25}$ but not all, studies. ${ }^{26}$ However, it should be noted that the extent of immobilization in these studies was substantial (complete immobilization, bed rest, or chronic spinal cord injury), whereas all participants in the current study were still mobile, although they had limited mobility from weakness and fatigue.

Our study has potential limitations. First, the number of participants was small, and the findings should be regarded as preliminary as the included participants may not be representative. Second, it could be argued that the different disease entities included in the IIMs group may have different pathological mechanisms. Although this may be correct, they also share a number of characteristics, some of which are likely to be relevant to the context of the current study and provide sufficient justification for grouping these entities for the purpose of this investigation. Another limitation is the fact that quadriceps strength was not tested in controls, but was only assumed to be normal. Last, due to a paucity of controls, we were not able to fully match for age. However, this is unlikely to have influenced the results, as SF does not appear to be affected by age. ${ }^{27}$

In conclusion, the results of this exploratory study suggest that force production of muscle fibers from patients with IIM are adversely affected by the disease process, and this could, at least partially, explain the weakness in these disorders. Further studies are required to elucidate the pathological mechanism responsible for the development of impaired contractility. In particular, the role of the pro-inflammatory cytokine TNF- $\alpha$ warrants further investigation in view of experimental animal data. Furthermore, studies with sufficient numbers of participants are required to determine contractility in each IIM subgroup.

\section{FUNDING INFORMATION}

National Research Foundation of South Africa (NRF Project: Muscle Contractility in GSD V), grant holder-linked bursary number: 81837 (to F.H. and T.K.); the Harry Crossley Foundation, grant number: SU-PT-15/09-000090 (to F.H.); Tim and Marilyn Noakes (postdoctoral fellowship to T.K.)

\section{CONFLICT OF INTEREST}

The authors declare no potential conflicts of interest.

\section{ETHICAL PUBLICATION STATEMENT}

We confirm that we have read the Journal's position on issues involved in ethical publication and affirm that this report is consistent with those guidelines.

\section{ORCID}

Franclo Henning (D) https://orcid.org/0000-0002-4006-8101

\section{REFERENCES}

1. Fink RH, Stephenson DG, Williams DA. Physiological properties of skinned fibres from normal and dystrophic (Duchenne) human muscle activated by Ca2+ and Sr2+. J Physiol. 1990;420:337-353.
2. Krivickas LS, Ansved T, Suh D, Frontera WR. Contractile properties of single muscle fibers in myotonic dystrophy. Muscle Nerve. 2000;23: 529-537.

3. Lassche S, Stienen GJ, Irving TC, et al. Sarcomeric dysfunction contributes to muscle weakness in facioscapulohumeral muscular dystrophy. Neurology. 2013;80:733-737.

4. Coley W, Rayavarapu S, Pandey GS, et al. The molecular basis of skeletal muscle weakness in a mouse model of inflammatory myopathy. Arthritis Rheum. 2012;64:3750-3759.

5. Dalakas MC. Muscle biopsy findings in inflammatory myopathies. Rheum Dis Clin N Am. 2002;28:779-798. vi.

6. Dalakas MC. Inflammatory muscle diseases. N Engl J Med. 2015;373: 393-394.

7. Dimachkie MM, Barohn RJ, Amato AA. Idiopathic inflammatory myopathies. Neurol Clin. 2014;32:595-628, vii.

8. Arahata K, Engel AG. Monoclonal antibody analysis of mononuclear cells in myopathies. I: Quantitation of subsets according to diagnosis and sites of accumulation and demonstration and counts of muscle fibers invaded by T cells. Ann Neurol. 1984;16:193-208.

9. Dalakas MC, Hohlfeld R. Polymyositis and dermatomyositis. Lancet. 2003;362:971-982.

10. Liang C, Needham M. Necrotizing autoimmune myopathy. Curr Opin Rheumatol. 2011;23:612-619.

11. Needham M, James I, Corbett A, et al. Sporadic inclusion body myositis: phenotypic variability and influence of HLA-DR3 in a cohort of 57 Australian cases. J Neurol Neurosurg Psychiatry. 2008;79: 1056-1060.

12. Askanas V, Engel WK, Nogalska A. Sporadic inclusion-body myositis: a degenerative muscle disease associated with aging, impaired muscle protein homeostasis and abnormal mitophagy. Biochim Biophys Acta. 1852;2015:633-643.

13. Benveniste O, Stenzel W, Hilton-Jones D, Sandri M, Boyer O, van Engelen BG. Amyloid deposits and inflammatory infiltrates in sporadic inclusion body myositis: the inflammatory egg comes before the degenerative chicken. Acta Neuropathol. 2015;129:611-624.

14. Kohn TA, Noakes TD. Lion (Panthera leo) and caracal (Caracal caracal) type IIx single muscle fibre force and power exceed that of trained humans. J Exp Biol. 2013;216:960-969.

15. Dada S, Henning F, Feldmann DC, Kohn TA. Baboon (Papio ursinus) single fibre contractile properties are similar to that of trained humans. J Muscle Res Cell Motil. 2018;39:189-199.

16. Godt RE, Maughan DW. Swelling of skinned muscle fibers of the frog. Experimental observations. Biophys J. 1977;19:103-116.

17. Kohn TA, Noakes TD. Lion (Panthera leo) and caracal (Caracal caracal) type IIx single muscle fibre force and power exceed that of trained humans. J Exper Biol. 2013;216:960-969.

18. Zong M, Bruton JD, Grundtman C, et al. TLR4 as receptor for HMGB1 induced muscle dysfunction in myositis. Ann Rheum Dis. 2013;72: 1390-1399.

19. Reid MB, Lannergren J, Westerblad H. Respiratory and limb muscle weakness induced by tumor necrosis factor-alpha: involvement of muscle myofilaments. Am J Respir Crit Care Med. 2002;166:479-484.

20. Wilcox PG, Wakai Y, Walley KR, Cooper DJ, Road J. Tumor necrosis factor alpha decreases in vivo diaphragm contractility in dogs. Am J Respir Crit Care Med. 1994;150:1368-1373.

21. Wilcox P, Milliken C, Bressler B. High-dose tumor necrosis factor alpha produces an impairment of hamster diaphragm contractility. Attenuation with a prostaglandin inhibitor. Am J Respir Crit Care Med. 1996;153:1611-1615.

22. Hardin BJ, Campbell KS, Smith JD, et al. TNF-alpha acts via TNFR1 and muscle-derived oxidants to depress myofibrillar force in murine skeletal muscle. J Appl Physiol. 2008;104:694-699.

23. D'Antona G, Pellegrino MA, Adami R, et al. The effect of ageing and immobilization on structure and function of human skeletal muscle fibres. J Physiol. 2003;552:499-511. 
24. Widrick JJ, Norenberg KM, Romatowski JG, et al. Force-velocitypower and force-pCa relationships of human soleus fibers after 17 days of bed rest. J Appl Physiol (1985). 1998;85:1949-1956.

25. Yamashita-Goto K, Okuyama R, Honda M, et al. Maximal and submaximal forces of slow fibers in human soleus after bed rest. $J$ Appl Physiol (1985). 2001;91:417-424.

26. Malisoux L, Jamart C, Delplace K, Nielens H, Francaux M, Theisen D. Effect of long-term muscle paralysis on human single fiber mechanics. J Appl Physiol (1985). 2007;102:340-349.

27. Trappe S, Gallagher P, Harber M, Carrithers J, Fluckey J, Trappe T. Single muscle fibre contractile properties in young and old men and women. J Physiol. 2013;552:47-58.

\section{SUPPORTING INFORMATION}

Additional supporting information may be found online in the Supporting Information section at the end of this article.

How to cite this article: Henning F, Kohn TA. An exploratory study of contractile force production in muscle fibers from patients with inflammatory myopathies. Muscle Nerve. 2020; 62:284-288. https://doi.org/10.1002/mus.26904 